\title{
Combinatorial auctions for electronic business
}

\author{
Y NARAHARI $^{1}$ and PANKAJ DAYAMA ${ }^{2}$ \\ ${ }^{1}$ Electronic Enterprises Lab, Computer Science and Automation, Indian Institute \\ of Science, Bangalore 560 012, India \\ ${ }^{2}$ GM India Science Lab, General Motors R \& D, ITPL Campus, \\ Bangalore 560 066, India \\ e-mail: hari@csa.iisc.ernet.in
}

\begin{abstract}
Combinatorial auctions (CAs) have recently generated significant interest as an automated mechanism for buying and selling bundles of goods. They are proving to be extremely useful in numerous e-business applications such as eselling, e-procurement, e-logistics, and B2B exchanges. In this article, we introduce combinatorial auctions and bring out important issues in the design of combinatorial auctions. We also highlight important contributions in current research in this area. This survey emphasizes combinatorial auctions as applied to electronic business situations.
\end{abstract}

Keywords. e-Business; combinatorial auctions; Vickrey-Clarke-Groves mechanisms; winner determination problem; bidding languages; multi-unit combinatorial auctions.

\section{Introduction}

Combinatorial auctions have emerged in recent times as an important mechanism, extremely useful in numerous e-business applications such as e-selling, e-procurement, e-logistics, supply chain formation, and B2B exchanges. The objectives of this paper are: (1) to introduce combinatorial auctions through illustrative examples and present the conceptual foundations; (2) bring out the main issues in the design of combinatorial auctions; and (3) to provide a glimpse of important contributions in the literature.

Several excellent survey papers have already appeared on combinatorial auctions. These include the exclusive surveys on combinatorial auctions by de Vries and Vohra [1] and by Pekec and Rothkopf [2], and more general surveys by Kalagnanam and Parkes [3], Shi [4], and Muller [5]. Cramton, Ausubel, and Steinberg [6] have recently brought out an edited volume containing expository and survey articles on all aspects of combinatorial auctions. Our current survey is intended to supplement and complement the others. In particular, the emphasis in our paper is on e-business applications.

A list of acronyms used is given at the end of the paper

References in this paper are not in journal format 


\subsection{Outline of the paper}

The rest of the paper is organized as follows.

Conceptual foundations ( $\S 2-3): \quad$ In $\S 2$, we present a classification of auctions as described by Kalagnanam and Parkes [3] and introduce combinatorial auctions. In $\S 3$, we consider an example of a combinatorial tennis auction and bring out important notions, concepts, and terminology in combinatorial auctions.

e-Business applications ( $\S 4-7): \quad$ Several applications of combinatorial auctions are first briefly looked at in $\S 4$. In $\S 5$, we describe the famous FCC (Federal Communications Commission) spectrum auction to illustrate a forward combinatorial auction and to explain the winner determination problem there. In $\S 6$, we present a logistics procurement auction case study involving home depot to illustrate a reverse combinatorial auction and the winner determination problem. In $\S 7$, we study a combinatorial procurement auction case study to illustrate a multi-unit combinatorial auction and the winner determination problem.

Design issues (§§8-10): In $\S 8$, we describe the challenges involved in the design of CAs. We first bring out the game theoretic and mechanism design theoretic view of design of CAs. We describe the important properties designers would expect from CAs and present the possibilities and impossibilities. We also touch upon the important issue of incentives. In $\S 9$, we focus our attention on the Generalized Vickrey Auction, which is a key mechanism in CAs satisfying many desirable properties. We then discuss, in $§ 10$, the computational complexity issues involved in implementing combinatorial auctions.

Current research (\$\$11-15): Sections 11-15 dwell on five important topics in CAs with a view to providing a glimpse of recent and current research there. Section 11 is on bidding languages; Section 12 on the winner determination problem; Section 13 on iterative CAs; Section 14 on multi-unit CAs; and $\S 15$ on combinatorial exchanges. In $\S 111-13$, our approach is to provide a fast-paced review with pointers to the literature (since there are excellent surveys available on these topics). In $\S \S 14-15$, we provide a slower paced review.

Conclusion (\$16-17): Section 16 presents a few practical implementation issues in deploying combinatorial auctions and $\S 17$ summarizes the contributions of this paper. We also provide a comprehensive bibliography.

\section{Auctions}

Auctions constitute a major class of economic mechanisms studied in microeconomics and game theory $[7,8]$. Classical mechanism design literature has delineated several useful properties for mechanisms such as efficiency, individual rationality, and budget balance, and incentive compatibility. The challenge in automating these mechanisms is to ensure computational tractability while retaining the desirable properties.

In this section, we briefly discuss a few important issues in auctions in order to build the necessary background for the subsequent discussion on combinatorial auctions.

\subsection{Types of auctions}

An auction is a mechanism to allocate a set of goods to a set of bidders on the basis of their bids. In a classical auction, the auctioneer wants to allocate a single item to a buyer among a 
group of bidders. There are four basic types of classical auctions prominently described in the literature [9-15]: English auction, Dutch auction, first price sealed-bid auction, and second price sealed-bid auction (also called the Vickrey auction). Auctions have evolved and grown far beyond these four types of mechanisms. Kalagnanam and Parkes [3] have suggested a framework for classifying auctions based on the requirements that need to be considered to set up an auction. These requirements fall into six categories [3].

(1) Resources: An auction involves a set of resources over which trade is to be conducted. The resource could be a single item or multiple items, with a single or multiple units of each item. Another common consideration is the number of attributes. In the case of multi-attribute items, the agents might need to specify the non-price attributes and some utility/scoring function to trade-off across these attributes.

(2) Market structure: An auction provides a mechanism for negotiation between buyers and sellers. In forward auctions a single seller sells resources to multiple buyers. In a reverse auctions, a single buyer attempts to source resources from multiple suppliers, as is common in procurement. Auctions with multiple buyers and sellers are called double auctions or exchanges.

(3) Preference structure: The preference structure of agents in an auction is important and impacts some of the other factors. The preferences define an agent's utility for different outcomes. For example, when negotiating over multiple units agents might indicate a decreasing marginal utility for additional units.

(4) Bid structure: The structure of the bids within the auction defines the flexibility with which agents can express their resource requirements. For a single unit, single item commodity, the bids required are simple statements of willingness to pay/accept. However, for multiunit identical items, bids need to specify price and quantity. This introduces the possibility for allowing volume discounts, where a bid defines the price as a function of the quantity. With multiple items, bids may specify all-or-nothing, both with price on a basket of items. In addition, agents might wish to provide several alternative bids but restrict the choice of bids.

(5) Matching supply to demand: A key aspect of auction is matching supply to demand, also referred to as market clearing, or winner determination. The main choice here is whether to use single-sourcing, in which pairs of buyers and sellers are matched, or multi-sourcing in which multiple suppliers can be matched with a single buyer, or viceversa.

(6) Information feedback: An auction protocol may be a direct mechanism or an indirect mechanism. In a direct mechanism such as a sealed bid auction, agents submit bids without receiving feedback, such as price signals, from the auction. In an indirect mechanism, such as an ascending-price auction, agents can adjust bids in response to information feedback from the auction. Feedback about the state of the auction is usually characterized by a price signal and a provisional allocation, and provides sufficient information about the bids of winning agents to enable an agent to redefine its bids.

The six dimensions above provide a matrix of choices that are available to set up the auction. The choices made for each of these dimensions will have a major impact on complexity of the analysis required to characterize the market structure that emerges, on the complexity on agents and the intermediary to implement the mechanism, and ultimately on the ability to design mechanisms that satisfy desirable economic and computational properties. 
In this paper, we focus our attention on multi-item auctions. In forward multi-item auctions (combinatorial selling), a bundle of different types of goods is available with the seller and buyers are interested in purchasing certain subsets of the goods. In reverse multi-item auctions (combinatorial procurement), a bundle of different types of goods is required by the buyer and several sellers (suppliers) are interested in selling subsets of the goods to the buyer. Other phrases for multi-item auctions are: combinatorial auctions, multi-object auctions, multigoods auctions, package auctions, and bundle auctions. We stick to the most popular usage, namely, combinatorial auctions. In multi-unit combinatorial auctions, there are multiple units of different items.

To sell a bundle of items, different approaches $[16,17]$ as shown below are possible: sequential auctions, parallel auctions, and combinatorial auctions. We describe these below.

Sequential auctions: Here the items are auctioned one at a time in some sequence. If the bidders have some preferences over combinations of items, it becomes difficult for them to submit bids as they do not know which items they will receive in later auctions. This can lead to inefficient allocations where bidders do not get their most preferred combinations.

Parallel auctions: Here the items are auctioned in parallel. However the bidder faces the same problem as one in sequential auctions. For example, when bidding for an item, the bidder does not know his valuation because it depends on which other items he will win which in turn is dependent upon the bids of other bidders.

Combinatorial auctions: This helps in overcoming the inefficiencies in allocations in sequential and parallel auctions. In combinatorial auctions (CAs), bidders can bid on combinations of items. For example, if $\mathrm{A}, \mathrm{B}$, and $\mathrm{C}$ are three different items, a bidder can place separate bids on seven possible combinations, namely, $\{A\},\{B\},\{C\},\{A, B\},\{B, C\},\{C, A\}$, and $\{\mathrm{A}, \mathrm{B}, \mathrm{C}\}$. In the case of $\mathrm{CAs}$, the value of an item a bidder wins depends on other items that he wins. The notions of complementarity and substitutability are very important in CAs.

Complementarity - Suppose an auctioneer is selling different goods. A bidder might be willing to pay more for the whole than the sum of what he is willing to pay for the parts. This property is called complementarity.

Substitutability - A bidder may be willing to pay for the whole only less than the sum of what he is willing to pay for parts. This is called substitutability. This is the case if the bidder has a limited budget or the goods are similar or interchangeable.

Because of these two notions, one cannot use sequential or parallel auctions as this might lead to inefficient allocations. Hence, combinatorial auctions provide a better choice.

\section{A simple example: An internet tennis auction}

To introduce some important notions and terminology, we discuss a stylized example. Let a seller (or auctioneer) be interested in auctioning the tennis rackets of Roger Federer and Maria Sharapova (winners of the 2004 All England Lawn Tennis Championships at Wimbledon). Let A and B be the rackets respectively, of Federer and Sharapova, with which they won the championship. The auctioneer invites bids for A and B through an internet auction. Let there be three buying agents (agent 1 , agent 2 , and agent 3 ) interested in buying one or both these tennis rackets. 
Table 1. Valuations for different bundles.

\begin{tabular}{lllr}
\hline & A & B & AB \\
\hline Agent 1 & 3 & 4 & 12 \\
Agent 2 & 5 & 5 & 5 \\
Agent 3 & 6 & 2 & 10 \\
\hline
\end{tabular}

\subsection{Terminology}

Let us denote the subsets $\{A\},\{B\},\{A, B\}$, through slight abuse of notation, by $\mathrm{A}, \mathrm{B}$, and $\mathrm{AB}$ respectively. We use the words package, bundle, and combination synonymously to mean a subset of items. A package bid is a bid from an agent for a package. A combinatorial bid is a collection of package bids from the same agent. Assume that the agents have valuations as shown in table 1 , for racket $\mathrm{A}$, racket $\mathrm{B}$, and for the combination of $\mathrm{A}$ and $\mathrm{B}$ (say, in thousands of euros). Valuation for a bundle of items represents the value of the bundle of items as perceived by that agent. The words valuation, value, willingness-to-pay, and reservation price are used interchangeably. In a sense, this is the maximum amount the agent is willing to pay for the bundle of items.

Note from table 1 that agent 1 values bundle $\mathrm{A}$ at 3 ; bundle $\mathrm{B}$ at 4 ; and bundle $\mathrm{AB}$ at 12 , which means he would prefer to win both A and B (that is, he likes Sharapova better than Federer but would love to possess both the tennis rackets rather than only one of them). He derives greater value out of the combination $A B$ than through A or B individually. Such a situation is called complementarity and the items A and B are said to be complementaries for agent 1 . Agent 2 has value 5 each for $\mathrm{A}$ alone, $\mathrm{B}$ alone, and $\mathrm{AB}$ combined. So she does not care which one she wins, she does not derive any extra benefit by winning both $\mathrm{A}$ and $\mathrm{B}$. In the case of agent 2 , the value for the combination $\mathrm{AB}$ is the maximum of the values for $\mathrm{A}$ and $\mathrm{B}$ and in this case, A and B are referred to as substitutes. Note that agent 3, like agent 1, has complementarity for the items A and B (though he likes Federer much more than Sharapova).

The values in table 1 satisfy the following condition: value of union of any two bundles is greater than or equal to the value of any individual bundle. This is called the free disposal property. This property means that disposing an item in a combination cannot increase the value of the combination. This is an important standard assumption in combinatorial auctions and we assume throughout this paper that this assumption is true. If, for example, $v(A)=4$, $v(B)=3$, and $v(A B)=2$, the free disposal condition is not satisfied.

When the selling agent invites bids, the buying agents typically would bid lower than their valuations. Rational agents will never bid above their valuations and they always bid so as to maximize their utilities. We assume that the utility function for each buying agent is of the quasi-linear form: $u_{i}(S)=v_{i}(S)-p_{i}(S)$ where $v_{i}(S)$ is the value that agent $i$ attaches to the bundle of items $S$ and $p_{i}(S)$ is the price the agent $i$ would pay to buy the bundle $S$ in case he is allocated the bundle (that is, in case he wins this bundle in the auction). The utility $u_{i}(S)$ is the utility derived by agent $i$ by winning the bundle $S$ through the auction. The terms payoff and reward are often used as synonyms for utility. A rational agent always follows the strategy of maximizing his utility while deciding what to bid for a bundle.

\subsection{A combinatorial auction for tennis rackets}

Let the three agents place their bids as shown in table 2 . Note immediately that all the bids are less than or equal to the respective valuations. Also, note that agent 2 has decided not 
Table 2. Bids from the agents for different bundles, say in lakhs of rupees.

\begin{tabular}{lccc}
\hline & A & B & AB \\
\hline Agent 1 & 2 & 3 & 7 \\
Agent 2 & 5 & 4 & $*$ \\
Agent 3 & 5 & 1 & 6 \\
\hline
\end{tabular}

to bid on the bundle $\mathrm{AB}$ (as shown by a "**" in the corresponding entry). Furthermore, all the bids are all-or-nothing type of bids. That is, when an agent bids for a bundle, either the entire bundle is allocated to the agent or nothing is allocated (partial allocation of a bundle is not allowed). Assume that these are XOR bids. That is, each agent wants to win exactly one of the package bids placed by him/her. For example, the combinatorial bid placed by agent 1 consists of three package bids $(\mathrm{A}, 2),(\mathrm{B}, 3)$, and $(\mathrm{AB}, 7)$ in XOR fashion. This would mean that he would like to be allocated exactly one of these three possibilities. If these bids were OR bids, then, more than one package bid from the same agent can be allocated. On receiving these bids, the seller is required to determine the allocation or select the winners. This is called the winner determination problem (WDP) or the allocation problem or the clearing problem. The WDP will require an objective function for determining the provisional winners. In this case, let us use maximum total bid as the objective function. With XOR bids, the winning allocation in the above will allocate bundle A to agent 3 and bundle B to agent 2 . Let us denote this allocation as $\{(3, \mathrm{~A}),(2, \mathrm{~B})\}$. The next important decision to take is how much the winning agents will pay. A straightforward way is to use pay-as-you-bid policy which means each winning bidder will pay what was bid. There are other non-trivial ways of doing it, which we will discuss later. Assuming pay-as-you-bid policy, the total revenue for the seller becomes $5+4=9$. Let $Z^{*}$ denote the total revenue to the seller, $V^{*}$ denote the sum of all values of the winning agents for the bundles won by them. We then see that $Z^{*}=9 ; V^{*}=6+5=11 ; u_{2}(B)=5-4=1 ; u_{3}(A)=6-5=1$, with rest of the payoffs equal to zero.

What we have described so far can be technically categorized as a first price, single round, sealed bid, forward combinatorial auction with XOR bids. It is called first price because the winners pay their bids. It is called a single round auction because bids are received exactly once and the final winners are computed based on these bids. In a multiple round auction (also called an iterative auction), there will be several such rounds of bidding and winner determination. It is called a forward auction because it corresponds to a selling scenario (with one seller and multiple buyers). A reverse auction on the other hand corresponds to a procurement scenario with one buyer and multiple sellers.

The winner determination problem for a general combinatorial auction is a non-trivial one, as we will see later. The payment determination problem also is an important and non-trivial problem.

If the bids are OR bids, instead of XOR bids, the winner determination problem will throw up two possible winning allocations, namely $\{(3, \mathrm{~A}),(2, \mathrm{~B})\}$ as before and one more allocation namely $\{(2, \mathrm{~A}),(2, \mathrm{~B})\}$. Multiple winning allocations are called ties and resolution of ties is an important problem in combinatorial auctions. Issues like fairness of allocation and transparency of allocation decisions arise in resolving these ties. For the second allocation $\{(2, \mathrm{~A}),(2, \mathrm{~B})\}$, we see that $Z^{*}=9 ; V^{*}=5+5=10 ; u_{2}(A)=5-5=0 ; u_{2}(B)=5-4=1$. 
Table 3. Another set of bids from the agents.

\begin{tabular}{lccc}
\hline & A & B & AB \\
\hline Agent 1 & $*$ & $*$ & 10 \\
Agent 2 & 5 & 4 & $*$ \\
Agent 3 & 5 & 1 & 6 \\
\hline
\end{tabular}

Note that the total value of this allocation, 10, is less than the total value of the previous allocation, which was 11. Maximizing the total value of allocation is an important criterion in combinatorial auctions. Such allocations are said to be allocatively efficient.

\subsection{Exposure problem and threshold problem}

If the above auction did not allow combinatorial bids, then it is possible that agent $1 \mathrm{might}$ end up winning A alone or B alone, which is not his most desired outcome. The problem of winning some but not all of a complementary collection of items in an auction without combinatorial bids is called the exposure problem. The word exposure is used because the agent is exposed to a possible loss if his bids include synergistic gains that might not be achieved. A properly designed combinatorial auction can completely overcome the exposure problem. For example, consider the bids from the agents to be as in table 3. Note that the bids of agent 2 and agent 3 are the same as in table 2, but agent 1 has a higher bid for $\mathrm{AB}$ and also has decided not to bid for A or B individually. In doing so, agent 1 could be prompted by the strong complementarity he has for the items A and B. With bids as in table 3, the winner is agent 1 and the exposure problem is solved. In case agent 1 bids only 8 for the bundle $A B$, then it will lose out, but because he has not bid for A or B individually, agent 1 does not face the exposure problem. If agent 1 decides to bid for A or B individually and the bids are as in table 4 , then the exposure problem resurfaces. This is because the winning allocation is now $\{(3, \mathrm{~A}),(1, \mathrm{~B})\}$. Agent 1 has succeeded in winning $\mathrm{B}$ but has lost $\mathrm{A}$ to agent 3 . Thus a combinatorial auction can often solve but cannot completely eliminate the exposure problem.

Though a combinatorial auction can often solve the exposure problem, it can introduce new problems such as the threshold problem. Allowing combinatorial bids may favour agents seeking larger packages. This is because agents seeking smaller packages may not have the incentive or the capability to outbid the heavy bids for the larger packages. For example, in table 4, agent 1 has placed a bid of 8 for $\mathrm{AB}$. Agent 2 will most likely lose out to agent 1 because it may not be able to win A or B individually unless she places high bids on these and, furthermore, is also supported by high bids on other items from other players.

The above scenarios also illustrate a distinct cooperative flavor that combinatorial auctions possess: A bundle cannot become a winning bid without a high enough bid on items which

Table 4. One more set of bids from the agents.

\begin{tabular}{llll}
\hline & A & B & AB \\
\hline Agent 1 & 3 & 4 & 8 \\
Agent 2 & 3 & 3 & $*$ \\
Agent 3 & 5 & 1 & 6 \\
\hline
\end{tabular}


are not in the bundle. In the above example (table 4), agent 3 is able to win item A because there is a high enough bid on the other item B (from agent 1).

\section{Some applications of combinatorial auctions}

Numerous applications have been reported in the literature for combinatorial auctions. We describe some of these applications briefly. Note in all the examples below that the problem is one of distributed resource allocation and bundles of resources are involved in bidding and allocation.

\subsection{FCC spectrum allocation}

FCC is the Federal Communications Commission, a federal agency in USA which allocates spectrum licenses. The problem here is to achieve an efficient (value maximizing) allocation of new spectrum licenses to wireless telephone companies [18-23]. The mobility of clients leads to synergistic values across geographically consistent license areas, for example the value for New York City, Philadelphia, and Washington DC might be expected to be much higher than the value of any one license by itself. It is required that all the licenses be allocated at the same time as some companies might value certain combination of licenses more than individual licenses. We describe this auction in more detail in $\$ 5$.

\subsection{Electronic procurement}

The combinatorial auction can be used for procuring direct or indirect materials. A buyer wishes to procure a bundle of items and sends an RFQ (request for quote) to several vendors. The vendors respond with quotes for subsets of items. The problem is to select the best mix of bids that minimizes the total cost of procuring the required bundle. This is one of the major application areas for combinatorial auctions since procuring a bundle of items rather than individual items can lead to savings in logistics cost, lead time reduction, and overall cost savings. See for example the following papers: [24-28].

\subsection{Bandwidth exchanges}

Slots of bandwidth are available of a fixed size and duration with public and private companies (sellers). Buyers (service providers or smaller companies) have values for bundles of slots. The allocation problem here is to assign combinations of bandwidth slots to buyers and match them with sellers so as to maximize the total surplus in the system (that is the total amount received from the buyers minus the total payments to be made to sellers) [29]. This problem leads to a combinatorial exchange.

\subsection{Logistics and transportation}

Procuring logistics or transportation services provides a natural application for combinatorial auctions since bundling is common and natural in logistics services. A logistics exchange consists of shippers (buyers) who would like to ship bundles of loads from several sources to several destinations and carriers (sellers) who specify the cost of shipping along the bundles of routes serviced by them. So, a logistics exchange also corresponds to a combinatorial exchange. For application of combinatorial bidding in logistics and transportation, see the papers: [30-35]. 


\subsection{Supply chain formation}

Consider the problem of determining the participants in a supply chain and in determining who will exchange what with whom and the rules of the exchanges. Automated, dynamic supply chain formation is currently an important problem and one of the approaches to solving this problem is based on combinatorial auctions [36]. The agents here are the potential participants in the supply chain. Each agent places bids on combinations of different resources in the supply chain. If the bidder does not get all components from the requested subset, then the transaction has no value to him.

\subsection{Distributed resource allocation}

In a manufacturing plant, a set of jobs is to be scheduled across a set of machines. Each job has some deadline and cost of delay and requires to be processed on several machines. The allocation problem here is to select the best mix of machine slots for individual jobs so as to minimize metrics like maximum tardiness or total delay, etc. Approaches based on combinatorial auctions have been suggested in [37-39].

Another application is in collaborative planning. Consider a system of robots [40] that wish to perform a set of tasks and have a joint goal to perform the tasks at as low a cost as possible. Suppose there are $n$ tasks to be performed and $m$ robots are available. Each robot requires a certain cost for performing a subset of tasks. The overall aim is to allocate subsets of tasks to robots so as to minimize the overall cost.

Many other resource allocation scenarios have been explored: for example, train scheduling [41], bus route allocation [42], and airport time slot allocation [43], and airspace resource allocation [44].

\subsection{Other applications}

A recent esoteric application is that of using combinatorial auctions in improving school meals [45]. Other interesting applications are in B2B negotiations $[46,47,36]$ and in planning of travel packages [48]. In travel package planning, the problem is to allocate flights, hotel rooms, and entertainment tickets to agents who have certain preferences over location, price, hotels, etc. Here, combinations are important because a hotel room without a flight ticket or an entertainment ticket has no value.

\section{Combinatorial auctions for spectrum allocation}

Selling frequency spectrum to telecommunication companies through on-line auctions was first attempted in New Zealand (1989) and in England (1990) [49]. Later, auctions were used for selling spectrum rights in Australia in 1993. These auctions failed to generate much revenue due to flaws in auction design. In 1994, the Federal Communications Commission (FCC) in USA conducted landmark auctions for frequency spectrum in which major telecommunication firms (long distance, local, cellular telephone companies, and cable television companies) participated [49]. This auction went through an elaborate design exercise in which many celebrated auction theorists provided their technical advice. The FCC divided the United States by geography and divided the spectrum by wavelength, resulting in 2500 licenses. There were 51 major trading areas, each of which had two large blocks of 30 megahertz spectrum. There were also 492 basic trading areas (which are subdivisions of major trading areas) each 
having one spectrum block of 20 megahertz and four spectrum blocks of 10 megahertz each. The total revenue to be generated by the auction was estimated as 10 billion US dollars.

Aggregation of licenses is an important factor for potential bidders in order to achieve efficiencies because of the following reasons [49]: (1) Firms which have several licenses can spread their fixed costs of technology acquisition and customer-base development. (2) Problems of interference at the boundaries of license areas imply that production-cost economies can be achieved by operating adjacent licenses. (3) Firms need to own enough licenses to cover reasonably large areas and hence offer roaming capability. (4) Each region contains several slices of the spectrum, hence a firm that fails to win one license may bid for another in its place. The first three reasons above correspond to complementarities while the fourth reason corresponds to substitutability. In general, different firms may like to bid for different license combinations. An auction for selling the 2500 licenses should therefore be flexible enough to enable the bidders to construct their own aggregations or combinations.

Several auction formats were discussed by auction theorists for realizing the spectrum auction objectives such as efficient outcome, preventing monopolization, and maximizing revenue. Instead of a conventional open auction, the FCC chose a simultaneous multiple round auction in which in each round the bidders submit sealed bids simultaneously on several individual licenses and the bids will be announced openly after every round, with a minimum bid increment. Such an auction, called a simultaneous ascending auction [19,23,22], allows bidders to take advantage of allowing any information revealed during the successive rounds and provides flexibility to aggregate their licenses. The auction was found to be largely successful.

One of the disadvantages of the simultaneous ascending auction is it does not allow combinatorial bids. For example, consider the case of three licenses A, B, and C. In the simultaneous ascending auction, there will then be three simultaneous auctions, the first one for A, the second one for $\mathrm{B}$, and the third one for $\mathrm{C}$. If a bidder wants either the combination $\{\mathrm{A}$, $\mathrm{B}\}$ or the combination $\{\mathrm{B}, \mathrm{C}\}$, then he is forced to bid in all the three auctions. Also, there is a possibility that he might win in zero, one, two, or three auctions, thus he might end up with undesirable combinations such as $\{A\},\{B\},\{C\},\{A, C\}$, or $\{A, B, C\}$, or he might even end up winning nothing. This would mean that the aggregation desired by the bidder may not be achievable. On the other hand, if combinatorial bidding is allowed, then the bidder bids on the just the combinations he desires, namely $\{\mathrm{A}, \mathrm{B}\}$ or $\{\mathrm{A}, \mathrm{C}\}$. For winning any of these two combinations (each of which provides him complementary benefits), he might be willing to bid a high price. The FCC auctions in the initial years did not allow combinatorial bidding, however more recently, combinatorial bids are allowed [50] making them even more efficient.

\subsection{A sealed bid combinatorial spectrum auction}

For the sake of illustration, we consider a stylized version of spectrum auctions, where there is only a single round. The combinatorial auction problem here can be stated as follows.

- An auctioneer (or a seller), in this case, the FCC, wants to sell a set $G=\left\{l_{1}, l_{2}, \ldots, l_{m}\right\}$ of $m$ distinct objects or goods or items (in this case, licenses).

- There is a set $N=\{1,2, \ldots, n\}$ of $n$ bidders (or buyers) who are interested in buying the entire set or some subsets of $G$.

- The auctioneer wants to maximize his revenue i.e. allocate the goods to the bidders with the highest bids.

- We assume XOR bidding language [51] is used i.e. each bidder receives only one subset. 
Table 5. Notation used for the FCC auction model.

\begin{tabular}{ll}
\hline$N=\{1,2, \ldots, n\}$ & Set of buying agents \\
$i \in N$ & Index for buying agents \\
$G=\left\{l_{1}, l_{2}, \ldots, l_{m}\right\}$ & Set of spectrum licenses up for sale \\
$k \in G$ & Index for spectrum licenses \\
$S \subseteq G$ & Any subset of $G$ \\
$b_{i}(S)$ & Bid of buyer $i$ for the subset $S$ \\
$y_{i}(S)$ & Boolean variable taking value 1 if buyer $i$ \\
& is allocated the set $S$ and 0 otherwise \\
$Z^{*}$ & Maximum sum of feasible mix of bids \\
\hline
\end{tabular}

Assume that the winners pay what they have bid. The seller wants to sell as much as possible while trying to maximize the revenue. The notation used here are given in table 5 . The winner determination problem can now be formulated as the following optimization problem.

$$
\begin{aligned}
& Z^{*}=\max \sum_{i \in N} \sum_{S \subseteq G} b_{i}(S) y_{i}(S) \\
& \text { s.t. } \sum_{S \ni k} \sum_{i \in N} y_{i}(S) \leq 1 \forall k \in G \\
& \sum_{S \subseteq G} y_{i}(S) \leq 1 \forall i \in N, \\
& y_{i}(S)=0,1 \forall S \subseteq G, \forall i \in N .
\end{aligned}
$$

The first constraint ensures that overlapping sets of goods are never assigned. The second constraint ensures that no bidder receives more than one subset (XOR bids).

The above problem is an instance of the weighted-set packing problem [1]. This is a wellknown NP-hard problem and in fact is inapproximable, which implies it is among the hardest NP-hard problems.

\section{Combinatorial auctions for logistics services at home depot}

This case study is reported in [27,52] and the description here is taken from [53]. Home Depot (HD) is the world's largest home improvement retailer with over 1000 stores and 37 distribution centers in United States, Canada, Puerto Rico and Chile and growing aggressively. The stores act as retail outlets as well as warehousing locations thereby combining economies of scale with a high level of customer service. Managing the logistics of this retailer involves coordinating over 7000 suppliers, numerous carriers, 1000 stores, and 37 distribution centers. A key component of this logistics effort is the transportation of over 40000 stock keeping units (SKU's) between entities in the supply chain using trucking companies. Traditionally, the bidding process for transportation contracts was completely manual wherein Home Depot would provide truckers with origin-destination zip codes for each pair of locations within its network and the aggregate demand forecasts for the pair. Based on this information, carriers would bid for each origin-destination pair that makes up a lane.

Such a bidding process has some obvious limitations: (1) carriers do not have good visibility to HD's network, (2) it did not allow carriers to bid on combinations of lanes to exploit 
potential synergies thereby limiting their ability to bid more aggressively on synergistic lanes, (3) the manual process is extremely inefficient. To achieve better efficiencies and effectiveness in transportation services, HD partnered with i2 Technologies, a leading provider of supply chain optimization software and a new flexible bidding mechanism deployed on the Internet was developed to allow carriers to bid for combinations of lanes as well as for individual lanes, thus allowing combinatorial bidding.

\subsection{A sealed bid reverse combinatorial auction}

In this problem, we have a single buyer and multiple sellers. The buyer tries to procure from the sellers at the least prices. The buyer has to procure at least the required set of lanes while minimizing the procurement cost. This reverse combinatorial auction problem can be stated as follows. The notation used here are given in table 6 .

- A buyer (in this case, home depot) wishes to buy a set $G=\left\{l_{1}, l_{2}, \ldots, l_{m}\right\}$ of distinct items (in this case, lanes).

- There is a set $N=\{1,2, \ldots, n\}$ of selling agents (in this case, carriers) who are interested in selling the entire set or some subsets of $G$.

- The buyer wants to minimize the procurement cost i.e. procure the goods from the bidders with minimum total cost.

- We assume XOR bidding language [51] is used, that is, the buyer buys at most one subset from any selling agent.

The winner determination problem can now be formulated as the following optimization problem.

$$
\begin{aligned}
& Z^{*}=\min \sum_{i \in N} \sum_{S \subseteq G} b_{i}(S) y_{i}(S), \\
& \text { s.t. } \sum_{S \ni k} \sum_{i \in N} y_{i}(S) \geq 1 \forall k \in G, \\
& \sum_{S \subseteq G} y_{i}(S) \leq 1 \forall i \in N, \\
& y_{i}(S)=0,1 \forall S \subseteq G, \forall i \in N .
\end{aligned}
$$

The first constraint ensures that at least the required set of goods is procured. The second constraint ensures that the buyer buys no more than one subset from any seller (XOR constraint).

Table 6. Notation used for the logistics combinatorial auction model.

$$
\begin{aligned}
& N=\{1,2, \ldots, n\} \\
& i \in N \\
& G=\left\{l_{1}, l_{2}, \ldots, l_{m}\right\} \\
& k \in G \\
& S \subseteq G \\
& b_{i}(S) \\
& y_{i}(S) \\
& Z^{*}
\end{aligned}
$$

Set of selling agents (carriers)

Index for seller agents

Set of items (lanes)

Index for items

Any subset of $G$

Bid of seller $i$ for subset $S$ of items

Boolean variable which takes value 1 if seller $i$

is allocated the subset $S$ and 0 otherwise

Minimum sum of feasible mix of bids 
The objective is to minimize the total cost. The winner determination problem in the reverse combinatorial auction above can be modelled as a weighted set covering problem (also known as minimum weighted set covering problem) [1]. This is a well-known NP-hard problem and in fact is inapproachable, which implies it is among the hardest NP-hard problems.

\section{Combinatorial procurement auctions at MARS Inc.}

This case study is reported in [26,54] and the description here is taken from [53]. The procurement activity at MARS Inc, a major global confectionary enterprise, described in [26], exhibits the following characteristics: (1) the supply pool is small for each category of material sometimes by necessity and sometimes by design, (2) a single buyer is responsible for large portfolios of items, (3) contracts are executed with many types of suppliers including private businesses, traded agricultural markets, monopolies, cartels, and governments, (4) negotiation and tendering are the most common procurement mechanisms, and (5) typical bids in these purchases include volume discounts and all-or-nothing bids. MARS realized that the process could be inefficient for several reasons: (1) competitive positions cannot be fully leveraged for price negotiations, (2) synergies or complementarities in supply conditions cannot be fully exploited in item by item negotiation, (3) disproportionate amount of time is spent determining quantities and prices, and (4) lack of transparency in award of contracts because of arbitrariness in the negotiation process. Automated auctions, generally seen as mechanisms that promote market competition and that make negotiations efficient, were proposed [26] to eliminate the limitations in the manual procurement process.

The MARS team working with researchers from the IBM T.J. Watson Research Center designed the auction mechanism that is currently used by buyers of MARS worldwide [26]. In order to meet with the business requirements outlined earlier and to accommodate complex bid structures, the team came up with an iterative auction design. An iterative auction, as explained by Cramton [55] and Parkes [56], are preferable to a single round sealed bid auction. In the case of MARS, an iterative auction has the following advantages: (1) it eliminates the need to completely specify the cost structure using bundled bids or volume discount bids which can result in exponentially large number of bids, (2) induces competition among suppliers as opposed to single shot bidding mechanisms, and (3) allows suppliers to correct their bids using information learned during the process. Each iteration proceeds in two stages: the first involves collecting a set of bids and finding the set(s) that minimize the cost of procurement. This is used as an input to the second stage where another optimization problem is solved whose objective is to minimize the sum of time stamps of the submitted bids with an additional constraint being that the cost of procurement is equal to the minimum cost obtained in the first stage of the iteration. By adopting such a solution process both the requirements of optimality and fairness are met.

The first step above is a reverse combinatorial auction problem, whose formulation is presented below. The formulation is from $[54,26]$. Table 7 provides the notation. $G$ is a set of items, where for each $k \in G$ there is a demand $d^{k}$. Each supplier $i \in N$ is allowed up to $M$ bids indexed by $j$. Associated with each bid $B_{i j}$ is a zero-one vector $a_{i j}^{k}, k=1, \ldots,|G|$ where $a_{i j}^{k}=1$ if $B_{i j}$ will supply the entire lot corresponding to item $k$, and zero otherwise. Associated with each bid $B_{i j}$ is price $p_{i j}$ at which the bidder is willing to supply the combination of items in the bid. A mixed integer programming (MIP) formulation can be written as follows.

$$
\min \sum_{i \in N} \sum_{j \in M} p_{i j} x_{i j}
$$


Table 7. Notation for combinatorial procurement.

\begin{tabular}{ll}
\hline$G$ & Set of items to be procured \\
$k \in G$ & Index for an item \\
$d^{k}$ & Number of units of item $k$ demanded \\
$N$ & Set of suppliers \\
$i \in N$ & Index for a supplier \\
$M$ & Set of bids allowed for a supplier \\
$j \in M$ & Index for a bid \\
$B_{i j}$ & Bid $j$ of supplier $i$ \\
$a_{i j}^{k}$ & 0-1 variable which takes value 1 iff $B_{i j}$ \\
& $\quad$ will supply the entire lot corresponding to item $k$ \\
$p_{i j}$ & Price associated with bid $B_{i j}$ \\
$W_{i, \min }$ & Minimum quantity that can be allocated to supplier $i$ \\
$W_{i, \max }$ & Maximum quantity that can be allocated to supplier $i$ \\
$x_{i j}$ & Decision variable that takes value 1 iff $B_{i j}$ is allocated \\
$y_{i}$ & Indicator variable that takes value 1 iff \\
$S_{\min }$ & supplier $i$ is allocated any lot \\
$S_{\max }$ & Minimum number of winners required \\
& Maximum number of winners allowed
\end{tabular}

subject to

$$
\begin{aligned}
& \sum_{i \in N} \sum_{j \in M} a_{i j}^{k} x_{i j} \geq 1, \quad \forall k \in G, \\
& x_{i j} \in\{0,1\} \forall i \in N, \quad \forall j \in M, \\
& W_{i, \min } y_{i} \leq \sum_{k \in G} \sum_{j \in M} a_{i j}^{k} d^{k} x_{i j} \forall i \in N, \\
& \sum_{k \in G} \sum_{j \in M} a_{i j}^{k} d^{k} x_{i j} \leq W_{i, \max } y_{i}, \quad \forall i \in N, \\
& \sum_{j \in M} x_{i j} \geq y_{i}, \quad \forall i \in N, \\
& S_{\min } \leq \sum_{i \in N} y_{i} \leq S_{\max }, \\
& y_{i} \in\{0,1\}, \quad \forall i \in N .
\end{aligned}
$$

$W_{i, \min }$ and $W_{i, \max }$ are the minimum and maximum quantities that can be allocated to any supplier $i$; Constraints (4) and (5) restrict the total allocation to any supplier to lie within $\left(W_{i, \min }, W_{i, \max }\right), y_{i}$ is an indicator variable that takes the value 1 if supplier $i$ is allocated any lot. $S_{\min }$ and $S_{\max }$ are respectively the minimum and maximum number of winners required for the allocation and constraint (7) restricts the winners to be within that range.

The above problem is a variant of a weighted set covering problem with side constraints. It is computationally quite hard and has been shown to be NP-hard [54].

\section{Design of combinatorial auctions}

The design of combinatorial auctions (auctions, in general) can be viewed as a problem of designing a mechanism that implements a social choice function. Designing a mechanism, in 
turn, can be viewed as a problem of designing a game with incomplete information having an equilibrium in which the required social choice function is implemented.

\subsection{Essentials of mechanism design}

Consider a set of agents $N=\{1,2, \ldots, n\}$ with agent $i$ having a type set $\Theta_{i}(i=1,2, \ldots, n)$. The type set of an agent represents the set of perceived values of an agent (also called private values). In the case of combinatorial auctions, the type of an agent refers to the valuation that the agent has for different bundles. Let $\Theta$ be the Cartesian product of all the type sets of all the agents (that is $\Theta$ is the set of all type profiles of the agents). Let $X$ be a set of outcomes. An outcome, in the context of combinatorial auctions, corresponds to assignment of bundles and payments to the bidders. A social choice function is a mapping from $\Theta$ to $X$ which associates an outcome with every type profile. In the context of auctions, a social choice function corresponds to a desirable way of producing outcomes from given type profiles. Let $S_{i}$ denote the action set of agent $i$, that is $S_{i}$ is the set of all actions that are available to an agent in a given situation. A strategy $s_{i}$ of an agent $i$ is a mapping from $\Theta_{i}$ to $S_{i}$. That is, a strategy maps each type of an agent to a specific action the agent will choose if it has that type. In an auction, a strategy corresponds to the bid the agent will place based on its observed type. Suppose $S$ is the Cartesian product of all the strategy sets. A mechanism is basically a tuple $\left(S_{1}, S_{2}, \ldots, S_{n}, g().\right)$, where $g$ is a mapping from $S$ to $X$. That is, $g($.$) maps each$ strategy profile into an outcome. Every mechanism can be associated with a game with incomplete information, which is called the game induced by the mechanism. For details, refer to $[57,58]$.

We say that a mechanism $\mu=\left(S_{1}, S_{2}, \ldots, S_{n}, g().\right)$ implements a social choice function $f$ if there is an equilibrium strategy profile $\left(s_{1}^{*}(),. s_{2}^{*}(),. \ldots, s_{n}^{*}().\right)$ of the game induced by $\mu$ such that $\left.g\left(s_{1}^{*}\left(\theta_{1}\right), s_{2}^{*}\left(\theta_{2}\right), \ldots, s_{n}^{*}\left(\theta_{n}\right)\right)=f\left(\theta_{1}, \theta_{2}, \ldots, \theta_{n}\right)\right)$ for all possible type profiles $\left(\theta_{1}, \theta_{2}, \ldots, \theta_{n}\right)$. That is, a mechanism implements a social choice function $f($.$) if there$ is an equilibrium of the game induced by the mechanism that yields the same outcomes as $f($.) for each possible profile of types. Depending on the type of equilibrium, we qualify the implementation. Two common types of implementations are dominant strategy implementation and Bayesian Nash implementation, corresponding respectively to dominant strategy equilibrium and Bayesian Nash equilibrium. For definitions of these, the reader is referred to [57]. The dominant strategy equilibrium is a strong and robust solution concept that ensures that the equilibrium strategy of each agent is best whatever the strategy profiles of the rest of the agents. The Bayesian Nash equilibrium is a weaker solution concept but is more easily achieved than a dominant strategy equilibrium.

A direct revelation mechanism corresponding to a social choice function $f($.$) is a mecha-$ nism of the form $\mu=\left(\Theta_{1}, \Theta_{2}, \ldots, \Theta_{n}, f().\right)$. That is, the strategy sets are the type sets itself and the outcome rule $g($.) is the social choice function itself. A social choice function is said to be incentive compatible in dominant strategies (or strategy proof or truthfully implementable in dominant strategies) if the direct revelation mechanism $\mu=\left(\Theta_{1}, \Theta_{2}, \ldots, \Theta_{n}, f().\right)$ implements $f($.) in a dominant strategy equilibrium where the equilibrium strategy of each agent is to report its true type. Similarly a social choice function is said to be Bayesian Nash incentive compatible if the direct revelation mechanism $\mu=\left(\Theta_{1}, \Theta_{2}, \ldots, \Theta_{n}, f().\right)$ implements $f($.) in a Bayesian Nash equilibrium where the equilibrium strategy of each agent is to report its true type. The revelation principle [57] states that if a function can be implemented in dominant strategies (or Bayesian Nash equilibrium), it can also be truthfully implemented in dominant strategies (or Bayesian Nash equilibrium). The revelation principle enables one to focus attention only on incentive compatible mechanisms. 
The mechanism design problem is to determine a mechanism that implements a "good" social choice function. Some desirable properties which are sought from a social choice function and hence from the implementing mechanism (and in the present case, from combinatorial auctions) are described below [57,58,2].

\subsection{Properties desired from a combinatorial auction mechanism}

Efficiency: A general criterion for evaluating a mechanism is Pareto efficiency, meaning that no agent could improve its allocation without making at least one other agent worse off. Another metric of efficiency is allocative efficiency which is achieved when the total value of all the winners is maximized. When allocative efficiency is achieved, the resources or items are allocated to the agents who value them most.

Individual rationality: A mechanism is individually rational if its allocations do not make any agent worse off than had the agent not participated in the mechanism. That is, every agent gains a non-negative utility by being a participant in the mechanism.

Budget balance: A mechanism is said to be weakly budget balanced if the the revenue to the auctioneer or the exchange is non-negative while it is said to be strongly budget balanced if this revenue is positive. Budget balance ensures that the auctioneer or the exchange does not make losses.

Incentive compatibility: A mechanism is incentive compatible if the agents optimize their expected utilities by bidding their true valuations for the goods. This is a desirable feature because an agent's decision depends only on its local information and it gains no advantage in expending effort to model other agents' valuations. It is desired that truthful bidding by the agents should lead to a well defined equilibrium such as a dominant strategy equilibrium or a Bayesian Nash equilibrium.

Solution stability: The solution of a mechanism is stable, if there is no subset of agents that could have done better, even if they came to an agreement outside the mechanism.

Revenue maximization or cost minimization: In an auction where a seller is auctioning a set of items, the seller would like to maximize total revenue earned. On the other hand, in a procurement auction, the buyer would like to procure at minimum cost. Given the difficulty of finding equilibrium strategies, designing cost minimizing or revenue maximizing auctions is not easy.

Low transaction costs: The buyer and sellers would like to minimize the costs of participating in auctions. Delay in concluding the auction is also a transaction cost. Also, the winner determination algorithm should be efficient and in fact should run in real-time if the auction is iterative.

Fairness: This influences willingness of bidders to participate in auctions. Winner determination algorithms, especially those based on heuristics, could lead to different sets of winners at different times (depending on the initial conditions set). Also, since there could be multiple optimal solutions, different sets of winners could be produced by different specific exact algorithms used. Bidders who lose out (they could have probably won if a different algorithm had been used) could end up feeling unfairly treated. A solution to this problem is to let the bidders know exactly which algorithms are employed for determining the winners. 


\subsection{Possibilities and impossibilities}

The properties of social choice functions or mechanisms listed above are quite conflicting and not all of them can be achieved simultaneously. There is a rich body of results in mechanism design theory dealing with what combinations of properties are possible and what are not possible to be achieved by an economic mechanism such as auctions. We informally state a few of these results below. It is beyond the scope of this article to state these rigorously and we attempt to provide the spirit of the results only. Interested reader should refer to the books by Mas-Colell, Whinston, and Green [57] and by Green and Laffont [59]. First, we look at some impossibilities.

- Hurwicz [60] showed that it is impossible to achieve allocative efficiency, weak budget balance, and individual rationality in a Bayesian Nash incentive compatible mechanism.

- Gibbard [61] and Satterthwaite [62] showed that only a very special class of social choice functions called dictatorial functions can be implemented truthfully in dominant strategies (in fairly general settings).

- According to Arrow [63], allocative efficiency and strong budget balance cannot be achieved in a dominant strategy equilibrium.

- Green and Laffont [59] showed that no allocatively and strategy proof mechanism can be safe from manipulation by coalitions, even in quasi-linear environments.

- Myerson and Satterthwaite [64] showed that no exchange (that is with multiple sellers and multiple buyers) can be efficient, budget balanced, and individual rational at the same time; this holds with or without incentive compatibility.

Fortunately, there are also some positive results about mechanisms that can be implemented.

- It was shown by Groves [65] and Clarke [66] that allocatively efficient and strategy proof mechanisms are possible if the utility functions are quasi-linear (that is, of the form utility $=$ value minus price). Clarke mechanisms are a special class of Groves mechanisms. The generalized Vickrey auction (GVA) [67] is a combinatorial auction version of Clarke's mechanisms while the Vickrey auction [68] (second price sealed bid auction of a single indivisible item) is a special case of GVA for non-combinatorial auctions. In fact, GVA satisfies four properties simultaneously: allocative efficiency, individual rationality, weak budget balance, and strategy proofness. All the mechanisms above are also commonly referred to as VCG (Vickrey-Clarke-Groves) mechanisms.

- It was shown by Arrow [63] and d'Aspremont and Gerard-Verat [69] that under quasilinear preferences, it is possible to have a mechanism (which is called the dAGVA mechanism) that is efficient, Bayesian Nash incentive compatible, strongly budget balanced, and individually rational in ex ante sense.

- It was shown by Myerson [70] that revenue maximization, individual rationality, and incentive compatibility can be achieved simultaneously.

- McAfee [71] showed that strategy proof double auctions are possible with weak budget balance.

- Krishna and Perry [72] have proved several positive results on existence of efficient mechanisms. For example, they have shown that the GVA mechanism maximizes the expected revenue amongst all efficient, Bayesian Nash incentive compatible, and individual rational mechanisms.

The above results provide a glimpse of what is possible and what is impossible in the design of mechanisms. For more details on these results, refer to [58,3,7,57,59]. Essentially, the 
above discussion shows that the design of combinatorial auctions involves fairly sophisticated techniques to be used. Even if a mechanism is implementable, the computational complexity involved in implementing it could make it unattractive, as we will see in $\S 9$.

\subsection{Incentive issues}

Incentives constitute an extremely important issue in auctions and we now present some issues related to incentives, based on the discussion in the papers by McAfee and McMillan [9] and Pekec and Rothkopf [2]. Informally, a mechanism describes any process that takes as inputs the bids of the agents and determines which bidders will be allocated the item(s) and how much payment is received by the winning bidders. A mechanism is incentive compatible if the mechanism is structured in a way that each bidder finds it optimal in some sense to report his valuation truthfully. An incentive compatible mechanism induces truth revelation by the bidders by designing the payoff structure in a way that it is in the best interests of the bidders to bid truthfully. The second price sealed bid auction or the Vickrey auction for a single unit of a single item has been shown to be incentive compatible [68] in dominant strategies. The generalized Vickrey auction is an example of an incentive compatible combinatorial auction mechanism [50,1]. Clarke mechanisms [66] and Groves mechanisms [65] provide a broader class of incentive compatible mechanisms. All these mechanisms are referred to as VCG (Vickrey-Clarke-Groves) mechanisms. These mechanisms induce truth revelation by providing a discount to each winning buying agent on his actual bid. This discount which is called the Vickrey Discount is actually the extent by which the total revenue to the seller is increased due to the presence of this bidder (marginal contribution of the bidder to the total revenue). If the agents are selling agents, then we have Vickrey Surplus which is the additional amount given to a selling agent over and above what he has quoted.

VCG mechanisms have very attractive properties. For example, the GVA mechanism already stated, is allocatively efficient, individual rational, weakly budget balanced, and incentive compatible. However these mechanisms are not commonly used for many reasons. The first reason is they are not revenue efficient because of the payment of Vickrey surpluses or Vickrey discounts. They are also subject to several kinds of manipulations and are unsustainable in realistic auction settings [2]. The third reason is the computation of Vickrey surpluses and Vickrey discounts involves solving as many NP-hard problems as the number of winning bidders. Recent work by Bikhchandani and Ostroy [73] has shown that only a linear program needs to be solved in some special instances to compute Vickrey surpluses and Vickrey payments (note that this is true only in some special instances).

\section{The generalized Vickrey auction}

A general method for implementing efficient and strategy proof mechanisms has been devised by Clarke and Groves $[66,65]$. When applied to combinatorial auctions, it generalizes the second price sealed bid auction of Vickrey [68] and is therefore called the generalized Vickrey auction (GVA). GVA is an important building block for design of combinatorial auctions. Several CA mechanisms are based on GVA. Examples of such mechanisms are discussed in [74-80].

In GVA, the allocation chosen maximizes the sum of the declared valuations of the bidders, each bidder receives a monetary amount that equals the sum of the declared valuations of all the winning bidders, and pays the auctioneer the sum of such valuations that would have been obtained if he had not participated in the auction. 
Table 8. Bids from agents for the GVA example.

\begin{tabular}{lccc}
\hline & A & B & AB \\
\hline Agent 1 & $*$ & $*$ & 2 \\
Agent 2 & 2 & $*$ & $*$ \\
Agent 3 & $*$ & 2 & $*$ \\
\hline
\end{tabular}

An example: Consider the stylized auction scenario discussed in $\S 4$ involving two items and three agents. Let the valuations be as in table 1 and the bids be as in table 2. Let the bids be XOR bids. The winning allocation here is: $\{(3, \mathrm{~A}),(2, \mathrm{~B})\}$, yielding a total bid value of $Z^{*}=5+4=9$ and a total value of winning bids as $V^{*}=6+5=11$. To determine the payments of the winning bidders, we compute the marginal contribution of the winners, namely agent 2 and agent 3 , to the auction. Let $Z_{-i}^{*}(i=1,2,3)$ be the total bid value of a winning allocation when agent $i$ (that is, the set of all bids of agent $i$ ) is removed from the auction. When agent 2 is removed, then the winning allocation is $\{(3, A),(1, B)\}$ with $Z_{-2}^{*}=5+3=8$. Therefore, the marginal contribution of agent 2 to the auction is $Z^{*}-Z_{-2}^{*}=9-8=1$. This is called the Vickrey discount of agent 2 . The VCG payment rule says that the payment of agent 2 is equal to her bid minus her Vickrey discount. Thus the Vickrey payment of agent 2 is $4-1=3$. Similarly when agent 3 is removed from the scene, the winning allocation is $\{(2, \mathrm{~A}),(1, \mathrm{~B})\}$ with $Z_{-3}^{*}=5+3=8$. Therefore, the marginal contribution of agent 3 to the auction is $Z^{*}-Z_{-3}^{*}=9-8=1$. This is the Vickrey discount for agent 3 . The Vickrey payment of agent 3 is $5-1=4$. Thus the total revenue generated for the seller is $3+4=7$, in contrast to $4+5=9$ in the pay-as-you-bid payment case.

Now let us say the agents bid exactly their true values (as in table 1). Then the winning allocation is $\{(1, \mathrm{AB})\} ; Z^{*}=12$; and $V^{*}=12$. If agent 1 is not present, then the winning allocation is $\{(3, \mathrm{~A}),(2, \mathrm{~B})\}$, with the corresponding values of total bid and total value as 11 and 11 , respectively. Thus the marginal contribution of agent 1 to the auction is $12-11=1$ and therefore agent 1 pays 11 to the seller. We can observe that both the revenue to the seller and also the total value to the winning buyer(s) are greater than in the previous case.

There are serious problems with GVA, however. Consider the bids from agents as shown in table 8 . In this case, the winning allocation is: $\{(2, \mathrm{~A}),(3, \mathrm{~B})\}$ with $Z^{*}=4$. We can compute and show that $Z_{-2}^{*}=2$ and $Z_{-3}^{*}=2$. Therefore, the marginal contribution of agent 2 and agent 3 to the auction is 2 each. Therefore, their Vickrey discounts will be 2 each, implying that their Vickrey payments are zero each! This is a serious problem which shows that the seller might end up with zero revenue if he uses GVA. Worse still, if agent 2 and agent 3 are both the false names of a single agent, then the auction itself is seriously manipulated! Yokoo, Sakurai, and Matsubura [81] study the effect of false name bids in combinatorial auctions in general and in GVA in particular.

\section{Computational complexity issues}

We have seen several possibility and impossibility results in the context of mechanism design. While every possibility result is good news, there could be still be challenges involved in actually implementing a mechanism that is possible. For example, we have seen that the GVA mechanism is allocatively efficient, strategy proof, individually rational for all agents, and weakly budget balanced. However, a major difficulty with GVA is the computational 
complexity involved in determining the allocation and the payments. Both the allocation and payment determination problems are NP-hard, being instances of the weighted set packing problem (in the case of forward GVA) or the weighted set covering problem (in the case of reverse GVA). In fact, if there are $n$ agents, then in the worst case, the payment determination will involve solving as many as $n$ NP-hard problems, so overall, as many as $(n+1) \mathrm{NP}$ hard problems will have to be solved. Moreover, approximately solving any one of these problems may destroy properties such as efficiency and strategy proofness of the mechanism. Fortunately, there are some special instances described by Bikhchandani and Ostroy [73] where the payment determination can be done by just solving a linear program.

The following discussion is from [58,3]. In a mechanism where resource allocation is done based on decentralized information, computations are involved at two levels: first, at the agent level and secondly at the mechanism level. The complexity questions involved are briefly indicated below.

\section{Complexity at the agent level}

- Strategic complexity: Must agents model other agents and solve game theoretic problems to compute an optimal strategy? For instance, in a sealed bid combinatorial procurement scenario, sellers will need to not only take their valuation of the bundles into consideration but also the bidding behavior of their competitors. This requires sophisticated bidding logic.

- Valuation complexity: How much computation is required to provide preference information within a mechanism? For instance, in a combinatorial procurement scenario where the items exhibit cost complementarities, estimating a bid for every possible permutation of the bundle of items requires exponential space and hence exponential time [56].

\section{Complexity at the mechanism level}

- Communication complexity: How much communication is required between agents and the mechanism to compute an outcome. For instance, in an iterative combinatorial auction, where individual valuations are revealed progressively in an iterative manner, the communication costs could be high if the auction were conducted in a distributed manner over space and/or time. Bidding languages have been developed to surmount this problem to some extent. We will discuss bidding languages in a following section.

- Winner determination complexity: How much computation is expected of the mechanism infrastructure to compute an outcome given the bid information of the agents. This is an extremely important question since we have already seen that the winner determination problem is often NP-hard. We discuss some aspects of the winner determination problem in a following section.

\section{Bidding languages}

In combinatorial auctions, bidder can bid on any subset of items. Since an exponential number of such subsets are possible, an expressive language could possibly help in expressing the bids efficiently. The simplest bidding language allows each bidder to bid a vector of bids corresponding to each possible subset of items. But the bid itself will become exponentially long. Thus the bidding language should be such that not only is it easy to express any vector of bids but also it should be simple to do manipulations with bids. These two conditions 
are conflicting, so one needs to strike a balance between them. Issues of bidding languages for CAs are discussed in detail by Nisan [51,82], Boutlier [83], and Sandholm [84]. A few common types of bidding languages are discussed below.

Atomic bids: Here the bidder submits a bid $(S, p)$ where $S$ is a subset of items and $p$ is the price he is willing to pay. Moreover, for subset $T$ if $S \subseteq T, v(T)=p$ else $v(T)=0$.

OR bids: Here a bidder can submit any number of atomic bids. It is assumed that he is willing to obtain any number of atomic bids for a price equal to the sum of their prices. OR bids can represent all bids that do not have substitutabilities. For example, a single item valuation on two items cannot be represented by OR-bids.

XOR bids: Here also a bidder can submit any number of atomic bids. It is assumed that he is prepared to obtain at most one of these atomic bids. XOR bids can represent all valuations. The size of a bid is the number of atomic bids in it.

OR-of-XOR bids: Here a bidder can submit any number of XOR bids. It is assumed that he is prepared to obtain any number of these bids, at a price equal to the sum of their respective prices. These bids are highly expressive.

XOR-of-OR bids: Here a bidder can submit any number of OR bids. It is assumed that he is prepared to obtain only one of these bids. These bids are also highly expressive.

OR bids with phantom items $\left(O R^{*}\right): \quad$ In this language the phantom item helps in expressing XOR bids as a variant of OR bids. Here bidders can introduce phantom items into bidding which will be helpful in expressing constraints without having any value to the bidder. Here each bidder can submit any number of atomic bids $\left(S_{l}, p_{l}\right)$ where $S_{l} \subseteq G \cup G_{i}, G$ is the set of items, $G_{i}$ is set of phantom items which only bidder $i$ can bid on and $p_{l}$ is maximum price bidder is willing to pay. It is assumed that the agent is willing to obtain any number of disjoint bids for sum of their respective prices. For example, an XOR bid $\left(S_{1}, p_{1}\right) X O R\left(S_{2}, p_{2}\right)$ can be represented as $\left(S_{1} \bigcup\{x\}, p_{1}\right) O R\left(S_{2} \bigcup\{x\}, p_{2}\right)$ where $x$ is a phantom item. It has been shown [51] that any valuation that can be represented by OR-of-XOR bids of size $S$, can be represented by $O R^{*}$ bids of size $S$, using at most $S$ dummy items. It is also shown [51] that any valuation that can be represented by XOR-of-OR bids of size $S$, can be represented by $O R^{*}$ bids of size $S$ using at most $S^{2}$ phantom items. Thus $O R^{*}$ bid language is at least as expressive as all other languages discussed.

For more details about bidding languages in combinatorial auctions, the reader is referred to the papers by Nisan [51,82], Boutlier [83], and Sandholm [84].

\section{Winner determination problem}

The allocation problem or the winner determination problem (WDP), i.e., determining the items that each bidder wins is not difficult in the case of non-combinatorial auctions. It would take $O(n m)$ time where $n$ is the number of bidders and $m$ is the number of items. But in the case of combinatorial auctions, as we have already seen, the WDP is much more complex. Recall that in forward CAs, the WDP turns out to be an instance of a weighted set packing problem whereas in reverse CAs, the WDP turns out to be an instance of a weighted set covering problem. Both these problems are known to be NP-hard [1]. In addition, if there are 
side constraints, like in the case of MARS combinatorial procurement problem (Section 7), the WDP becomes even harder to solve. It is important to solve the WDP exactly because only exact solutions may guarantee desirable properties such as efficiency and strategy proofness (such as in GVA). Further, these exact solutions would be required in quick time if the auction is iterative and provisional allocations and payments need to be announced in successive rounds.

The WDP is by far the most researched issue in combinatorial auction. The algorithms proposed fall into two classes: exact algorithms [85-89,51,90,16,91,92,84,93,94] and approximate algorithms [86,95-97]. Sandholm [84] provides a good summary of winner determination algorithms for combinatorial auctions. Naive methods are first surveyed followed by sophisticated search algorithms using a host of bid ordering heuristics. Wide experimentation is carried out on different kinds of problems to analyze the efficiency of the presented algorithms. In the second part of the paper, the authors consider expressive bidding languages such as XOR and OR-of-XOR using which both complementarity and substitutability of items can be captured. It is shown how these languages enable the use of VCG mechanisms to construct truthful combinatorial auctions. The search algorithms are extended for these bidding languages as well. Sandholm, Suri, Gilpin, and Levine [98] describe a fast algorithm which they call $\mathrm{CABOB}$ (combinatorial auction branch on bids). CABOB is a search algorithm that uses decomposition techniques, upper bounding, lower bounding, structural properties, and a host of bid ordering heuristics. It performs better than a standard optimization solver such as CPLEX 7.0 in most situations.

There are a few special cases where the WDP can be solved exactly using polynomial time algorithms $[87,1,99]$. For example, Sven de Vries and Vohra [1] look at polynomially solvable instances of the WDP in terms of the structure of the constraint matrix: totally unimodular matrices, balanced matrices, and perfect matrices. A matrix is said to be totally unimodular if the determinant of every square submatrix is 0,1 , or -1 . A $0-1$ matrix is called balanced if it has no submatrix of odd order with exactly two 1's in each row and column. A matrix is said to be perfect if it is the vertex-clique adjacency matrix of a perfect graph. In all these cases, it is shown that the WDP can be solved as a linear program. There are many other interesting special cases discussed in $[87,99]$.

Approximate algorithms have also emerged as a major approach to solving the allocation problem in CAs. We do not discuss them here but refer the reader to the following papers: $[74,100,96,95,51,86,93]$.

The WDP for a multi-unit combinatorial auction where there are multiple units of each item is obviously even more difficult than the WDP for single unit CAs. The case of multi-unit CAs will be taken up in a following section.

\section{Iterative combinatorial auctions}

Iterative CAs have emerged as a major approach, especially in e-business applications. Since the Internet enables enhanced communication capabilities, iterative auctions can be effectively implemented. In the iterative approach, there are multiple rounds of bidding and allocation and the problem is solved in an iterative and incremental way. Iterative CAs are attractive to bidders because they learn about their rivals' valuations through the bidding process, which could help them to adjust their own bids. Also, the iterative format provides enough opportunities for them to correct any bidding blunders they might commit in earlier rounds. On the other hand, iterative auctions open up the space for strategizing and bidders may collude. Care is required therefore in revealing only what is needed during the iterative process. 
There is now an extensive body of literature on iterative auctions. We refer the reader to the excellent surveys in $[58,3,101]$ and to the research papers $[102,103,50,73,104-108,76-$ 78,80,109-119].

The theory of linear programming duality provides an important foundation for these approaches. The papers by Bikhchandani and Ostroy [73,120] provide a treatment of the the use of duality theory in designing iterative combinatorial auction mechanisms. The paper by Bertsekas [121] describes a set of iterative auction algorithms for solving network flow problems. Many of the iterative auction mechanisms in the literature (for example, iBundle [58]) are based on these auction algorithms discussed by Bertsekas.

\section{Multi-unit combinatorial auctions}

In multi-unit combinatorial auctions, each item has multiple instances or units. In a forward multi-unit CA, the auctioneer wishes to sell a bundle that consists of multiple units of different types of items and the buying agents submit multi-unit combinatorial bids where they may specify bids for different subsets (which could be multi-sets). The winner determination problem is obviously more challenging than in the case of single unit CAs. There are several recent algorithms proposed here: Bikhchandani and Ostroy [73], Brown, Pearson, and Shoham [122,123], Gonen and Lehmann [124,125], Sandholm [84], and Bartal et al [126]. We describe a few of these below.

Dang and Jennings [127] consider multi-unit combinatorial auctions where the bids are piece-wise linear curves. Partial bids are allowed. Maximizing the revenue of the auctioneer is the objective. In the case of multi-unit, single-item auctions, the complexity of the clearing algorithm is $O\left(n(K+1)^{n}\right)$ where $n$ is the number of bidders and $K$ is an upper bound on the number of segments of the piecewise linear pricing functions. In the case of multi-unit, multiitem auctions, the clearing algorithm has complexity $O\left(m n(K+1)^{n}\right)$ where $m$ is the number of items. Note that the clearing algorithms have exponential complexity in the number of bids. Also, truth revelation and other game theoretic considerations are not taken into account.

Sandholm, Suri, Gilpin, and Levine [128] consider six different versions of combinatorial mechanisms: forward auctions, reverse auctions, and exchanges, each with single items or multiple items. In addition, they also consider the nature of free disposal (with free disposal, without free disposal), which makes it 12 types. (Free disposal means that buyers are willing to accept more than their requirement and sellers are willing to accept less than their requirement. If free disposal is not allowed, sellers have to sell everything and buyers cannot accept anything beyond what they have bid for).

The results are the following.

- Single unit or multi-unit combinatorial auction with free disposal: The decision version of both problems is NP-complete. The problem cannot even be approximated to within $n^{1-\epsilon}$ in polynomial time. The results are true even for integer prices and integer units. Finding a feasible solution is trivial.

- Single unit or multi-unit combinatorial reverse auction with free disposal: The decision version of both problems is NP-complete. The results are true even for integer prices and integer units. Finding a feasible solution is trivial. In the single unit case, the problem can be approximated to within $1+\log K$ factor of the optimum where $K$ is the largest number of items that any one bid contains.

- Single unit or multi-unit combinatorial exchange with free disposal: The decision version of both problems is NP-complete. The problem cannot even be approximated to within 
$n^{1-\epsilon}$ in polynomial time. The results are true even for integer prices and integer units. Finding a feasible solution is trivial.

- If free disposal is not allowed, even finding a feasible solution is NP-complete for all the categories of markets mentioned above. Also, the winner determination problems in all the cases above are not even approximable.

Sandholm and Suri [129] present a fast, sophisticated search algorithm, BOB (BranchOn-Bids), for the winner determination problem in combinatorial auctions. The algorithm improves upon earlier ones proposed by the authors and can also be applied to multi-unit combinatorial auctions and to multi-unit combinatorial exchanges. To make the algorithm fast, the authors employ several means such as structural improvements, faster data structures, solving tractable special cases, and search node-based optimizations.

Leyton-Brown, Shoham, Tennenboltz [123] present an algorithm called CAMUS (combinatorial auction multi-unit search) to compute winners in a general multi-unit combinatorial auction. The method uses a branch and bound technique in conjunction with an upper bounding function that is tailored specifically to the multi-unit combinatorial auction problem. The authors also introduce dynamic programming techniques to efficiently handle multi-unit, single-item bids. A preprocessing technique, a caching technique for already found solutions, and heuristics for determining search orderings provide additional efficiency to the winner determination algorithm.

Gonen and Lehmann [124] investigate the use of branch and bound heuristics for solving the winner determination problem in multi-unit combinatorial auctions. It is shown that the problem is equivalent to the weighted multi-set packing problem which is not only NPhard but cannot even be approximated within $N^{\frac{1}{2-\epsilon}}$ in polynomial time for any $\epsilon \geq 0$. The best approximation ratio and an ordering criterion that provides it are derived. The authors investigate two issues in using branch-and-bound methodology: (1) finding the best upper bounds (2) deciding the order in which bids are explored. Different methods for computing upper bounds are suggested: linear programming, projections, and fast heuristics. Different criteria are suggested for choosing the most promising bid.

Gonen and Lehmann [125] use a branch-and-bound methodology to solve the winner determination problem in multi-unit combinatorial auctions. They use linear programming to compute a good upper bound to the optimal solution and present a way of economizing the number of calls to the LP routine. The performance of different bid ordering heuristics is compared in this framework. It is shown that the gap between the lower bound provided by greedy heuristics and upper bound provided by LP is quite small, thus resulting in extensive pruning of the search space.

\section{Combinatorial exchanges}

In combinatorial exchanges, we have multiple buyers and multiple sellers, with combinatorial bids. The allocation and pricing problems here are one dimension more complex than combinatorial auctions. There are several recent efforts here: Parkes, Kalagnanam, and Eso [58,130], Smith et al [131], Kothari et al [132], Biswas and Narahari [79,80], Chu and Shen [133], Chu, Li, and Shen [134], and Jain and Varaiya [135,136]. We briefly describe some of these contributions.

Parkes, Kalagnanam, and Eso [58,130] consider sealed bid, single-shot combinatorial exchanges where the buying agents and selling agents place combinatorial bids. Motivated 
by impossibility theorems in exchange design, the authors use budget balance and individual rationality as hard constraints and design an exchange that is fairly efficient and fairly truthful. Vickrey payments are viewed as an assignment of discounts to agents after the exchange clears. Budget balance is achieved as long as the exchange distributes no more than the available surplus when the exchange clears. The pricing problem is solved as an optimization problem that minimizes distance to Vickrey discounts. Several different distance functions are considered, leading to different payment schemes. Experimentation and theoretical analysis suggest a simple threshold payment scheme which provides discounts to agents with payments greater than a threshold distance to their Vickrey payments.

The paper by Kothari, Sandholm, and Suri [132] considers a very general type of exchange: multi-unit, multi-item combinatorial exchange, where the bids are in the form of a bundle containing multiple units of multiple items with an associated price. Clearing such an exchange is obviously intractable and the paper considers the special case where acceptance of partial bids is allowed. Let $k$ be the number of items. The following are the results of this paper.

- If the objective is maximization of total surplus and at most $k$ partial bids are accepted, then the exchange can be cleared in polynomial time.

- If the objective is maximization of trade volume (total number of units sold) and at most $k+1$ partial bids are accepted, then the exchange can be cleared in polynomial time.

- The above results are used to develop $O(n \log n)$ algorithms for clearing single item, multi-unit exchanges, where $n$ is the number of combinatorial bids. This is done for both surplus maximization and trade volume maximization.

The paper by Smith, Sandholm, and Simmons [131] presents a design for an market maker to construct and clear a combinatorial exchange for trading single units of multiple items. The exchange uses preference elicitation by which the market maker elicits a reduced number of bids, only enough to prove that a particular allocation is optimal in the sense of maximizing welfare over all bidders. Preference elicitation uses structure inherent in bidder preferences to intelligently elicit only relevant bids, while still ensuring that the exchange finds a welfare maximizing outcome. The proposed method also includes item discovery that constructs the exchange even as preferences are being elicited.

In his doctoral dissertation, Biswas [80] has come up with interesting iterative mechanisms for combinatorial exchanges. First, he has developed an iterative auction mechanism to solve the combinatorial exchange problem. In each iteration the combinatorial exchange problem is decomposed into computationally simpler combinatorial forward and reverse auctions. Next, Lagrangian relaxation is used to develop two iterative tâtonnement mechanisms to solve the combinatorial exchange problem. Finally, combinatorial exchanges are studied where the demand can be aggregated or the supply can be aggregated. For such exchanges, iterative Dutch auction schemes are proposed to solve the exchange problem.

\section{Practical implementation issues}

It is clear that auctions in general and combinatorial auctions in particular have wide scope in e-business applications. In this paper, we have seen three real-world applications: FCC spectrum auctions ( $\$ 5)$; logistics services procurement by Home Depot (\$6); and direct materials procurement by MARS ( $\$ 7)$. Several other applications have also been reported: airspace resource allocation [44]; planning and allocation of truckloads in transportation [34]; allocation of bus routes [42]; transportation services for Sears logistics [31] conducted by Net 
Exchange (www . nex . com); school meals planning [45] etc. Corporate procurement appears to be a major application area for combinatorial auctions. Pekec and Rothkopf [2] have mentioned in their survey that a range of companies have started using combinatorial auctions: CombineNet.com, TradeExtensions.com, Logistics.com, etc. Leading solution providers such as Ariba, i2 Technologies, SAP, Free Markets, etc. mention combinatorial auctions as a mechanism implemented in their solutions. Going by popular press and Internet reports, it is clear that many companies have already successfully deployed combinatorial auctions in their eprocurement and e-business operations. However due to the proprietary nature of such information, there is little open documentation in this area.

Given the proven potential of combinatorial auctions in e-business, it is important to be aware of key issues in successful deployment of CAs. Recent advances in combinatorial auction technology and theory which when properly deployed, can result in significant additional cost savings and increase in profits. Some of these advances can be algorithmically integrated into e-business solutions leading ultimately to cost minimization and profit maximization for companies. There are however, several challenges involved.

- Though combinatorial auctions are much more effective than traditional auctions in naturally modelling e-business situations, there are many situations where non-combinatorial auctions have been preferred for various reasons (see for example, early versions of FCC auctions [18]). Thus, before deploying CAs, non-combinatorial mechanisms also will have to be considered as an option.

- Bidding languages have to be properly chosen, balancing between expressiveness and user-friendliness.

- Computational complexity (Section 10) is a major issue in deploying CAs, so the algorithms have to be chosen carefully. Numerous algorithms that have been proposed in the literature in the last five years for solving different types of CA problems. This entails an informed choice of algorithms based on the exact requirements.

- Approximate algorithms for solving the allocation problem provide an attractive alternative, however approximate solutions may destroy desirable properties such as allocative efficiency and strategy proofness.

- Several industry applications have been demonstrated for CAs mostly in the eprocurement and e-logistics contexts. These solutions have however been developed by specific industries for specific situations. CAs are not yet commodified by e-business vendors, because of the custom requirements entailed by different e-business situations.

- Combinatorial auctions with enhanced features such as multi-units and multi-attributes will add significant value to e-business solutions, however, the winner determination problem in these situations is extremely computation-intensive.

- Collusion by bidders, formation of coalitions, false name bids etc. can severely affect the success of a combinatorial auction and they need to be looked at in more detail.

\section{Summary}

Combinatorial auctions (CAs) provide a mechanism for combinatorial bidding by participating agents enabling the agents to express their preferences for bundles of items rather than individual items. They are proving to be extremely useful in numerous e-business applications, especially, in e-procurement, e-logistics, and B2B exchanges. In this article, we have:

- introduced the conceptual foundations of combinatorial auctions, 
- presented representative e-business applications with formulations of the winner determination problem,

- brought out key issues in the design of combinatorial auctions, and

- provided a glimpse of current research in several topics in combinatorial auctions.

As already stated in the introduction, several survey papers have already appeared on combinatorial auctions. These include the exclusive surveys on combinatorial auctions by de Vries and Vohra [1] and by Pekec and Rothkopf [2], and more general surveys by Kalagnanam and Parkes [3], Shi [4], and Muller [5]. Cramton, Ausubel, and Steinberg [6] have recently brought out an edited volume containing expository and research articles on all aspects of combinatorial auctions. This book also contains an extremely useful glossary of terms. We hope our survey will complement the other existing surveys. We would urge the reader to consult the above survey papers and the research papers cited in this paper to explore further on this exciting topic. Researchers and graduate students who would like to pursue algorithm development and experimental work in this area can peruse the data sets available from [137].

This research is supported by a research grant IDEAS (Intelligent Digital Economy Algorithms) from Intel India Development Center, Bangalore, India. The initiative and interest shown by Mr. Gopalan Oppiliappan, Ms Vijaya Kumari, and Ms Shubhra Aurita Roy have been instrumental in taking up this important research project, and their support is gratefully acknowledged. We also would like to deeply appreciate the collaboration with the Manufacturing Systems Research Laboratory, GM R \& D, Warren, Michigan. In particular, we thank Dr. Jeffrey D. Tew, Dr. Datta Kulkarni, Dr. Charlie Rosa and Dr. Earnest Foster of the above laboratory for useful discussions.

\section{Acronyms}

LP Linear program

ILP integer linear program

CA combinatorial auction

GVA generalized Vickrey auction

VCG Vickrey-Clarke-Groves (mechanisms)

WDP winner determination problem

FCC federal communications commission

\section{References *}

1. S. de Vries and R. V. Vohra. Combinatorial auctions: A survey. INFORMS Journal of Computing, 15(1), 2003

2. A. Pekec and H.M. Rothkopf. Combinatorial auction design. Management Science, 49: 14851503,2003

3. J. Kalagnanam and D. Parkes. Auctions, bidding, and exchange design. In (eds) D. Simchi-Levi, D. Wu, and Shen, Supply Chain Analysis in the eBusiness Area. Kluwer Academic Publishers, 2005

4. Y. Shi. Combinatorial auctions : Mathematical formulations. International Journal of Information Technology and Decision Making, 2(1), 2003

*References in this list are not in journal format 
5. R. Muller. Auctions - the big winner among trading mechanisms for the internet economy. Technical report, MERIT - Infonomics Memorandum Maastricht, Netherlands, 2001

6. P. Cramton, Y. Shoham, and R. Steinberg (eds). Combinatorial Auctions. The MIT Press, Cambridge, MA, 2005

7. Vijay Krishna. Auction Theory. Academic Press, 2002

8. P. Milgrom. Putting Auction Theory to Work. Cambridge University Press, 2004

9. R.P. McAfee and J. McMillan. Auctions and bidding. Journal of Economic Literature, 25:699_ 738, 1987

10. P. Milgrom. Auctions and bidding: a primer. Journal of Economic Perspectives, 3(3):3-22, 1989

11. J.H. Kagel. Auctions: A survey of experimental research. In (eds) J.H. Kagel and A.E. Roth, The Handbook of Experimental Economics, pages 501-587. Princeton University Press, Princeton, 1995

12. E. Wolfstetter. Auctions: An introduction. Economic Surveys, 10:367-421, 1996

13. P. Klemperer. Auction theory: a guide to the literature. Journal of Economic Surveys, pages 227-286, 1999

14. M. Herschlag and R. Zwick. Internet auctions-a popular and professional literature review. Electronic Commerce, 1(2):161-186, 2000

15. P. Klemperer. Auctions: Theory and Practice. Online Book, www.paulklemperer.org, 2004

16. T. Sandholm. An algorithm for optimal winner determination in combinatorial auctions. In Proceedings of International Joint Conference on Artificial Intelligence (IJCAI-99, pages 542_ 547, 1999

17. P. Cramton, Y. Shoham, and R. Steinberg. Introduction to combinatorial auctions. In P. Cramton, Y. Shoham, and R. Steinberg, editors, Combinatorial Auctions. The MIT Press, Cambridge, MA, 2005

18. J. McMillan. Selling spectrum rights. Technical report, University of California, San Diego, 1995

19. P.C. Cramton. The FCC spectrum auction: an early assessment. Journal of Economics and Management Strategy, 6(3):431-495, 1997

20. P. Cramton and J. Schwartz. Collusive bidding: Lessons from the FCC spectrum auctions. Journal of Regulatory Economics, 17, 2000

21. M. M. Bykowsky, R. J. Cull, and J. O. Ledyard. Mutually destructive bidding: The FCC auction design problem. Journal of Regulatory Economics, 2000

22. L.M. Ausubel and P. Milgrom. Ascending proxy auctions. In P. Cramton, Y. Shoham, and R. Steinberg, editors, Combinatorial Auctions. The MIT Press, Cambridge, MA, 2005

23. P. Cramton. Simultaneous ascending auctions. In P. Cramton, Y. Shoham, and R. Steinberg, editors, Combinatorial Auctions. The MIT Press, Cambridge, MA, 2005

24. M. Pravin Kumar. Private e-marketplace for procurement: Architecture and algorithms. Technical Report M E Project Report, Dept. of Computer Science \& Automation, Indian Institute of Science, Bangalore, 2001

25. J. Gallien and L. M. Wein. Design and analysis of a smart market for industrial procurement. Technical report, Operations Research Center, MIT, Cambridge, MA, 2000

26. G. Hohner, J. Rich, E. Ng, G. Reid, A.J. Davenport, J. R. Kalagnanam, S.H. Lee, and C. An. Combinatorial and quantity discount procurement auctions provide benefits to mars, incorporated and to its suppliers. Interfaces, 33(1):23-35, 2003

27. W. Elmaghraby and P. Keskinocak. Combinatorial auctions in procurement. Technical report, School of Industrial and Systems Engineering, Georgia Institute of Technology, 2002

28. M. Bichler, A. Davenport, G. Hohner, and J. Kalagnanam. Industrial procurement auctions. In P. Cramton, Y. Shoham, and R. Steinberg, editors, Combinatorial Auctions. The MIT Press, Cambridge, MA, 2005

29. M. Eso, J. Kalagnanam, L. Ladanyi, and Y.G. Li. Winner determination in bandwidth exchanges. Technical report, IBM TJ Watson Research Center, 2001 
30. J. Song and A. C. Regan. Combinatorial auctions for transportation since procurement : The carrier perspective. Technical report, Institute of Transportation Studies, University of California, Irvine, 2002

31. J.O. Ledyard, M. Olson, D. Porter, J.A. Swanson, and D.P Torma. The first use of a combined value auction for transportation services. Interfaces, 32(5):4-12, 2002

32. Y. Narahari and S. Biswas. An iterative auction mechanism for combinatorial logistics exchanges. In The 9th International Symposium on Logistics. ISL-2004, Bangalore, July 2004

33. C. Caplice and Y. Sheffi. Theory and practice of optimization-based bidding for motor carriers transport services. Journal of Business Logistics, 24(2):109-128, 2003

34. C. Caplice and Y. Sheffi. Combinatorial auctions for truckload transportation. In P. Cramton, Y. Shoham, and R. Steinberg, editors, Combinatorial Auctions. The MIT Press, Cambridge, MA, 2005

35. Y. Sheffi. Combinatorial auctions in the procurement of transportation services. Interfaces, 34 , 2004.

36. W. E. Walsh, M. P. Wellman, and Fredrik Ygge. Combinatorial auctions for supply chain formation. In Proceedings of ACM Conference on Electronic Commerce (EC-00), pages 260269, 2000

37. E. Kutanoglu and S. D. Wu. On combinatorial auctions and Lagrangean relaxation for distributed resource scheduling. IIE Transactions, 31(9):813-826, September 1999.

38. P. R. Wurman. Market structure and multidimensional auction design for computational economies. Technical report, Doctoral Dissertation, University of Michigan, Ann Arbor, MI, 1999

39. M.P. Wellman, W.E. Walsh, P.R. Wurman, and J.K. MacKie-Mason. Auction protocols for decentralized scheduling. Games and Economic Behavior, 35:271-303, 2001

40. L. Hunsberger and B. J. Gross. A combinatorial auction for collaborative planning. In Proceedings International Conference on Multi-Agent Systems (ICMAS-00, pages 151-158, 2000

41. D. C. Parkes and L. H. Ungar. An auction-based method for decentralized train scheduling. In Proceedings of International Conference on Autonomous Agents (AGENTS-01), 2001

42. E. Cantillon and M. Pesendorfer. Auctioning bus routes:The London experience. In P. Cramton, Y. Shoham, and R. Steinberg, editors, Combinatorial Auctions. The MIT Press, Cambridge, MA, 2005

43. S. J. Rassentiand V. L. Smith and R. L. Bulfin. A combinatorial mechanism for airport time slot allocation. Bell Journal of Economics, 13:402-417, 1982

44. M.O. Ball, G.L. Donahue, and K. Hoffman. Auctions for safe, efficient, and equitable allocation of airspace resources. In P. Cramton, Y. Shoham, and R. Steinberg, editors, Combinatorial Auctions. The MIT Press, Cambridge, MA, 2005

45. R. Epstein, L. Henriquez, J. Catalan, G.Y. Weintraub, and C. Martinez. A combinatorial auction improves school meals in chile. Interfaces, 32(6):1-14, 2002

46. J. L. Jones. Incompletely specified combinatorial auction: An alternative allocation mechanism for business-to-business negotiations (Ph.D. thesis), University of Florida, Gainsville, FL, 2000

47. J. L. Jones and G. J. Koehler. Multi-criteria combinatorial auction: A B2B allocation mechanism for substitute goods. Proceedings of the Americas Conference on Information Systems, 2000

48. A. Greewald and J. Boyan. Bidding algorithms for simultaneous auctions: A case study. In Proceedings of ACM Conference on Electronic Commerce (EC-01), 2001

49. J. McMillan. Selling spectrum rights. Journal of Economic Perspectives, pages 145-162, 1994

50. L.M. Ausubel and P.R. Milgrom. Ascending auctions with package bidding. Frontiers of Theoretical Economics, 1(1):1-50, 2002

51. N. Nisan. Bidding and allocation in combinatorial auctions. In Proceedings of ACM Conference on Electronic Commerce (EC-00), 2000

52. W. Elmaghraby and P. Keskinocak. Technology for transportation bidding at the home depot. In C. Billington, T. Harrison, H. Lee, and J. Neale, editors, Practice of Supply Chain Management: Where Theory and Practice Converge. Kluwer Academic Publishers, 2003 
53. T.S. Chandrashekar, Y. Narahari, C. H. Rosa, D. Kulkarni, and J. D. Tew. Auction based mechanisms for automated industrial procurement. Technical report, Department of Computer Science and Automation, Indian Institute of Science, Bangalore http://lcm.csa.iisc.ernet.in/hari/, 2004

54. A. Davenport and J. Kalagnanam. Price negotiations for direct procurement. Research Report RC 22078, IBM Research, Yorktown Heights, NJ, USA, 2001

55. P.C. Cramton. Ascending auction. European Economic Review, 42(3):745-756, 1998

56. D. Parkes. Auction design with costly preference elicitation. Annals of Mathematics and Artificial Intelligence, 2005

57. A. Mas-Collel, M. D. Whinston, and J. R. Green. Microeconomic Theory. Oxford University Press, 1995

58. D. Parkes. Iterative Combinatorial Auctions: Achieving Economic and Computational Effciency. PhD thesis, Department of Computer and Information Science, University of Pennsylvania, Philadelphia, PA, May 2001

59. J.R. Grren and J.J. Laffont. Incentives in Public Decision Making. North-Holland, Amsterdam, 1979

60. L. Hurwicz. On informationally decentralized systems. In C. McGuire and Roy Radner, editors, Decision and Organization: A Volume in Honor of Jacob Marchak. North-Holland, Amsterdam, 1972

61. A. Gibbard. Manipulation of voting schemes. Econometrica, 41:587-601, 1973

62. M.A. Satterthwaite. Strategy proofness and arrow's conditions: Existence and correspondence theorems for voting procedures and social welfare function. Journal of Economic Theory, 10:187-217, 1975

63. K. Arrow. The property rights doctrine and demand revelation under incomplete information revelation. In (ed.) M. Boskin, Economics and Human Welfare. Academic Press, New York, 1979.

64. R.B. Myerson and M. A. Satterthwaite. Efficient mechanisms for bilateral trading. Journal of Economic Theory, 28:265-283, 1983

65. T. Groves. Incentives in teams. Econometrica, 41:617-631, 1973

66. E. Clarke. Multi-part pricing of public goods. Public Choice, 11:17-23, 1971

67. H. Varian. Economic mechanism design for computerized agents. Technical Report, University of California, Berkeley, 2000

68. W. Vickrey. Counter speculation, auctions, and competitive sealed tender. Journal of Finance, 16:8-37, 1961

69. C. d'Aspremont and L.A. Gerard-Verat. Incentives and incomplete information. Journal of Public Economics, 11:25-45, 1979

70. R. B. Myerson. Optimal auction design. Mathematics of Operations Research, 6:58-73, 1981

71. J. McAfee. A dominant strategy double auction. The Journal of Economic Theory, 56:434-450, 1992

72. V. Krishna and M. Perry. Efficient mechanism design. Technical report, Department of Economics, Pennsylvania State University, University Park, PA, 1998

73. S. Bikchandani and J.M. Ostroy. The package assignment model. Journal of Economic Theory, 107:377-406, 2002

74. D. Lehmann, L. O'Callaghan, and Y. Shoham. Truth revelation in rapid, approximately efficient combinatorial auctions. In Proceedings of ACM Conference on Electronic Commerce (EC-99, pages 96-102, 1999

75. N. Nisan and A. Ronen. Computationally feasible veg-based mechanisms. International Journal of Electronic Commerce, 2000

76. D. C. Parkes. An iterative generalized Vickrey auction: Strategy-proofness without complete revelation. In Proceedings of AAAI Spring Symposium on Game Theoretic and Decision Theoretic Agents, 2001 
77. S. Biswas and Y. Narahari. Iterative reverse dutch auction for electronic procurement. In Proceedings of the International Conference on Electronic Commerce Research, ICECR-5, Montreal, Canada, December 2002

78. S. Biswas and Y. Narahari. Iterative combinatorial dutch auctions. Annals of Mathematics and Artificial Intelligence (Special issue), 2005 (to appear)

79. S. Biswas and Y. Narahari. Design of an iterative auction for combinatorial exchanges. Technical report, Department of Computer Science and Automation, Indian Institute of Science, Bangalore, http://lcm.csa.iisc.ernet.in/hari/, 2004

80. Shantanu Biswas. Design of Iterative Mechanisms for Combinatorial Auctions and Exchanges. $\mathrm{PhD}$ thesis, Department of Computer Science and Automation, Indian Institute of Science, Bangalore, April 2004

81. Y. Sakurai M. Yokoo and S. Matsubara. Robust combinatorial auction protocol against falsename bids. In Proceedings of National Conference on Artificial Intelligence (AAAI-2000, number 17, pages 110-116, 2000

82. N. Nisan. Bidding languages for combinatorial auctions. In P. Cramton, Y. Shoham, and R. Steinberg, editors, Combinatorial Auctions. The MIT Press, Cambridge, MA, 2005

83. C. Boutlier and H. Hoos. Bidding languages for combinatorial auctions. In Proceedings of the 17th International Joint Conference on Artificial Intelligence, 2001

84. T. Sandholm. An algorithm for optimal winner determination in combinatorial auctions. Artificial Intelligence, 135(1):1-54, 2002

85. A. Anderson, M. Tenhunen, and F. Ygge. Integer programming for combinatorial auction winner determination. In Proceedings of International Conference on Multi-Agent Systems (ICMAS-00), 2000

86. Y. Fujishima, K. Leyton-Brown, and Y. Shoham. Taming the computational complexity of combinatorial auctions: Optimal and approximate approaches. In Proceedings of International Joint Conference on Artificial Intelligence (IJCAI-99), pages 548-553, 1999

87. M. H. RothKopf, A. P. Pekec, and R. M. Harstad. Computationally manageable combinatorial auctions. Management Science, 44(8):1131-1147, 1998

88. R. C. Holte. Combinatorial auctions, knapsack problems, and hill-climbing search. In Canadian Conference on AI, pages 57-66, 2001

89. H. H. Hoos and C. Boutilier. Solving combinatorial auctions using stochastic local search. In Proceedings of National Conference on Artificial Intelligence (AAAI-O0), 2000

90. D. C. Parkes. Optimal auction design for agents with hard valuation problems. In Proceedings of Workshop on Agent Mediated Electronic Commerce (AmEC-99), 1999

91. T. Sandholm and S. Suri. Improved algorithms for optimal winner determination in combinatorial auctions and generalizations. In Proceedings of National Conference on Artificial Intelligence (AAAI-2000), pages 90-97, 2000

92. M. Tennenholtz. Some tractable combinatorial auctions. In Proceedings of National Conference on Artificial Intelligence (AAAI-00), 2000

93. D. Lehmann, R. Muller, and T. Sandholm. The winner determination problem. In (eds) P. Cramton, Y. Shoham, and R. Steinberg, Combinatorial Auctions. The MIT Press, Cambridge, MA, 2005

94. T. Sandholm. Optimal winner determination algorithms. In (eds) P. Cramton, Y. Shoham, and R. Steinberg, Combinatorial Auctions. The MIT Press, Cambridge, MA, 2005

95. Y. Sakurai, M. Yokoo, and K. Kamel. An efficient approximate algorithm for winner determination in combinatorial auctions. In Proceedings of ACM Conference on Electronic Commerce (EC-00), p 30-37, 2000

96. E. Zurel and N. Nisan. An efficient approximate allocation algorithm for combinatorial auctions. In Proceedings of ACM Conference on Electronic Commerce (EC-01), p 125-136, 2001

97. R. Kastner, C. Hsieh, M. Potkonjak, and M. Sarrafzadeh. On the sensitivity of incremental algorithms for combinatorial auctions, In IEEE Int. Workshop on Advanced Issues of e-Commerce and Web-based Information Systems (WECWIS), 2002 
98. T. Sandholm, S. Suri, A. Gilpin, and D. Levine. Cabob: A fast optimal algorithm for combinatorial auctions. In International Joint Conference on Artificial Intelligence (IJCAI-01), Seattle, Washington, USA, p 1102-1108, 2001

99. R. Muller. Tractable cases of the winner determination problems. In (eds) P. Cramton, Y. Shoham, and R. Steinberg, Combinatorial Auctions. The MIT Press, Cambridge, MA, 2005

100. B. Lehmann, D. Lehmann, and N. Nisan. Combinatorial auctions with decreasing marginal utilities. In Proceedings of ACM Conference on Electronic Commerce (EC-01), p 18-28, 2001

101. D.C. Parkes. Iterative combinatorial auctions. In (eds) P. Cramton, Y. Shoham, and R. Steinberg, Combinatorial Auctions. The MIT Press, Cambridge, MA, 2005

102. L. M. Ausubel. An efficient ascending-bid auction for multiple objects. Technical report, Department of Economics, University of Maryland, College Park, MD, 1997

103. L. M. Ausubel. An efficient dynamic auction for heterogeneous commodities. Technical report, Department of Economics, University of Maryland, College Park, MD, 2002

104. C. DeMartini, A. M. Kwasnica, J. O. Ledyard, and D. Porter. A new and improved design for multi-object iterative auctions. Technical report, California Institute of Technology, Pasadena, CA, 1998

105. P. Milgrom. Putting auction theory to work: Ascending auctions with package bidding. Journal of Political Economy, 108(2):245-272, 2000

106. D. C. Parkes. ibundle: An efficient ascending price bundle auction. In Proceedings of ACM Conference on Electronic Commerce (EC-99), p 148-157, 2000

107. D. C. Parkes and L. H. Ungar. Iterative combinatorial auctions: Theory and practice. In Proceedings of National Conference on Artificial Intelligence (AAAI-00, p 74-81, 2000

108. D. C. Parkes and L. H. Ungar. Preventing strategic manipulation in iterative auctions: Proxy agents and price-adjustment. In Proceedings of National Conference on Artificial Intelligence (AAAI-00), p 82-89, 2000

109. P. R. Wurman and M. P. Wellman. Akba: A progressive, anonymous-price combinatorial auction. In Proceedings of ACM Conference on Electronic Commerce (EC-00), p 21-29, 2000

110. P. R. Wurman and M. P. Wellman. Equilibrium prices in bundle auctions. In Proceedings of AAAI-99 Workshop on Artificial Intelligence for Electronic Commerce, p 56-61, 1999

111. F. Gul and E. Stachetti. Walrasian equilibrium without complementarities. Technical report, Michigan Center for Research on Economic and Social Theory, Ann Arbor, MD, 1997

112. F. Gul and E. Stachetti. Walrasian equilibrium with gross substitutes. Journal of Economic Theory, 87:95-124, 1999

113. F. Gul and E. Stachetti. The english auction with differentiated commodities. Journal of Economic Theory, 92:66-95, 2000

114. V. Bansal and R. Garg. Efficiency and price discovery in multi-item auctions. ACM SigEcom Exchanges, 2(1), 2001

115. R. Garg and V. Bansal. Simultaneous online independent auctions with discrete bid increments. Electronic Commerce Research, 2004

116. Debasis Mishra and David Parkes. A simple ascending generalized vickrey auction. Technical Report Harvard EconCS Technical Report, Harvard University, Cambridge, MA, 2004

117. Debasis Mishra and David Parkes. A vickrey-dutch clinching auction. Technical Report Harvard EconCS Technical Report, Harvard University, Cambridge, MA, 2004

118. Debasis Mishra and David Parkes. Ascending price vickrey auctions using primal-dual algorithms. Technical Report Harvard EconCS, Technical Report, Harvard University, 2004

119. Debasis Mishra and David Parkes. Multi-item vickrey-dutch auction for unit-demand preferences. Technical Report Harvard EconCS Technical Report, Harvard University, 2004

120. S. Bikhchandani and J.M. Ostroy. From the assignment model to combinatorial auctions. In P. Cramton, Y. Shoham, and R. Steinberg, editors, Combinatorial Auctions. The MIT Press, Cambridge, MA, 2005

121. D.P. Bertsekas. Auction algorithms for network flow problems: A tutorial introduction. Computational Optimization and Applications, 1:7-66, 1992 
122. K. Leyton-Brown, M. Pearson, and Y. Shoham. Towards a universal test suite for combinatorial auction algorithms. In Proceedings of ACM Conference on Electronic Commerce (EC-O0), p 66-76, 2000

123. K. Leyton-Brown, Y. Shoham, and M. Tennenboltz. An algorithm for multi-unit combinatorial auctions. In Proceedings of National Conference on Artificial Intelligence (AAAI-00), 2000

124. R. Gonen and D. Lehmann. Optimal solutions for multi-unit combinatorial auctions: Branch and bound heuristics. In Proceedings of ACM Conference on Electronic Commerce (EC-O0), p 13-20, 2000

125. R. Gonen and D. Lehmann. Linear programming helps solving large multi-unit combinatorial auctions, http://citeseer.nj.nec.com/gonen01linear.html

126. Y. Bartal, R. Gonen, and N. Nisan. Incentive compatible multiunit combinatorial auctions. In Proceedings of Dagstuhl Seminar on Electronic Market Design, 2002

127. V.D. Dang and N.R. Jennings. Optimal clearing algorithms for multi-unit single-item and multiunit combinatorial auctions with demand-supply function bidding. Technical report, Dept of Electronics and Computer Science, University of Southampton, UK, 2003

128. T. Sandholm, S. Suri, A. Gilpin, and D. Levine. Winner determination in combinatorial auction generalizations. In Proceedings of International Conference on Autonomous Agents (Agents2001), 2001

129. T. Sandholm and S. Suri. Bob: Improved winner determination in combinatorial auctions and generalizations. Artificial Intelligence, 145:33-58, 2003

130. D. C. Parkes, J. Kalagnanam, and Marta Eso. Achieving budget-balance with Vickrey-based payment schemes in combinatorial exchanges. Technical Report RC 22218 W0110-065, IBM Research Report, 2001

131. T. Smith, T. Sandholm, and R. Simmons. Constructing and clearing combinatorial exchanges using preference elicitation. In Proceedings of National Conference on Artificial Intelligence (AAAI-02), 2002

132. A. Kothari, T. Sandholm, and S. Suri. Solving combinatorial exchanges : Optimality via a few partial bids. In Proceedings of National Conference on Artificial Intelligence (AAAI-02), 2002

133. L. Chu and Z.J. Shen. Dominant strategy double auction with pair-related cost. Technical report, Department of Industrial Engineering, University of Florida, Gainsville, Florida, USA, 2004

134. L. Chu, Y. Li, and Z.J. Shen. Double auction mechanisms for supply chain formation problems. Technical report, Department of Industrial Engineering, University of Florida, Gainsville, Florida, USA, 2004

135. R. Jain and P.P. Varaiya. Combinatorial bandwidth exchange: mechanism design and analysis. Comminications in Information and Systems, 3(4):305-324, September 2004

136. R. Jain and P.P. Varaiya. Efficient bandwidth allocation through auctions. In Proceedings of IEEE Infocom-2005, 2005

137. K. Leyton-Brown and Y. Shoham. A test suite for combinatorial auctions. In (eds) P. Cramton, Y. Shoham, and R. Steinberg, Combinatorial Auctions. The MIT Press, Cambridge, MA, 2005 